\title{
Acquired double-barrel oesophagus in epidermolysis bullosa dystrophica
}

\author{
R B WARREN, T F C S WARNER, E F GILBERT, AND J R PELLET \\ From the Departments of Pathology and Surgery, University of Wisconsin-Madison, \\ Clinical Science Center, Madison, Wisconsin, USA
}

ABSTRACT An unusual case of epidermolysis bullosa dystrophica with extensive stenosis, high perforation, and dissection of the oesophagus forming a "double-barrel" structure is described.을 Gastric epithelium found in the upper oesophagus is thought to be of metaplastic origin and caused by repeated minor trauma with repair.

The most common non-dermatological complication encountered in epidermolysis bullosa dystrophica is bulla formation, ulceration, and scarring with stricture of the oesophagus. ${ }^{1}$ Perforation of the oesophagus is very rare, and formation of a double-barrel oesophagus has not been reported. Here we present a case of epidermolysis bullosa dystrophica in which an oesophageal stricture with ulceration, dissection, perforation, and a double-barrel deformity developed late in the course of the disease. There was also extensive replacement of oesophageal epithelium by gastric mucosa.

\section{Case report}

A 34-year-old white woman was admitted to hospital with sudden haemoptysis associated with chest pain.

Shortly after birth, the patient's skin was noted to be friable and easily traumatised, resulting in indolent ulcers with scar formation. Throughout her childhood, she appeared underdeveloped for her age and was chronically anaemic. Numerous bullae and excoriations developed on her hands, feet, forearms, legs, elbows, knees, and back (fig 1). All nails were lost. Several successful autografts were performed, but eventually fusion of digits and toes by scar tissue occurred and progressed relentlessly to "mitten deformities" (fig 2). Most of her teeth were extracted because of an oedematous, friable, and hypertrophic gingiva. By the age of 13 years she could rot open her mouth more

Address for reprint requests: Dr RB Warren, Department of Pathology, University of Wisconsin-Madison, Clinical Science Center, 600 Highland Avenue, Madison, Wisconsin 53792, USA. than two centimetres. At age $17 \mathrm{yr}$, an oesophageal stricture was found on barium swallow, and another study, nine years later, showed no change. Her diet consisted of pureed food. At $\bar{D}$ age $30 \mathrm{yr}$, a benign verrucous lesion appeared on $\frac{\partial}{8}$ the left foot. It recurred two years later at $\stackrel{\mathbb{Q}}{2}$ which time a diagnosis of low grade squamous $\overrightarrow{0}$ cell carcinoma was made. There have been 3 several recurrences despite treatment. A sister, three years older than the patient had been? similarly affected, and died at 27 years of squamous cell carcinoma. The parents are first 을 cousins. No other living or dead relative is

known to be affected by the disease.
On the day of admission, the patient had attempted to force down solid food. Vomiting $\delta$ and haemoptysis developed, associated with severe upper throat pain, which later radiated to을 the chest and subxiphoid area. On physical $>$ examination no abnormalities were detected in the lungs apart from diminution of respiratory $N_{\circ}$ excursion and severe pain on deep breathing. The abdomen was rigid with guarding. Scaling 0 erythematous lesions were present over thew distal extremities, chest, and neck. Alopecia areata was present. Mediastinal and subdiaphrag 0 matic air were detected on the chest radiograph. At barium swallow examination, contrast medium $\stackrel{?}{+}$ deviated sharply to the right at the carinal level $\frac{0}{\circ}$ while a thin wisp of contrast coursed down to $\underset{\vec{\Phi}}{\vec{d}}$ the stomach.

A thoracotomy was performed the next day. $\stackrel{\mathbb{Q}}{\stackrel{2}{2}}$ Exploration revealed free cloudy fluid withing the pleural space and an oedematous media- $O$ stinum with crepitus. At approximately the midportion of the oesophagus, a tiny perforation was found. The oesophagus was? 


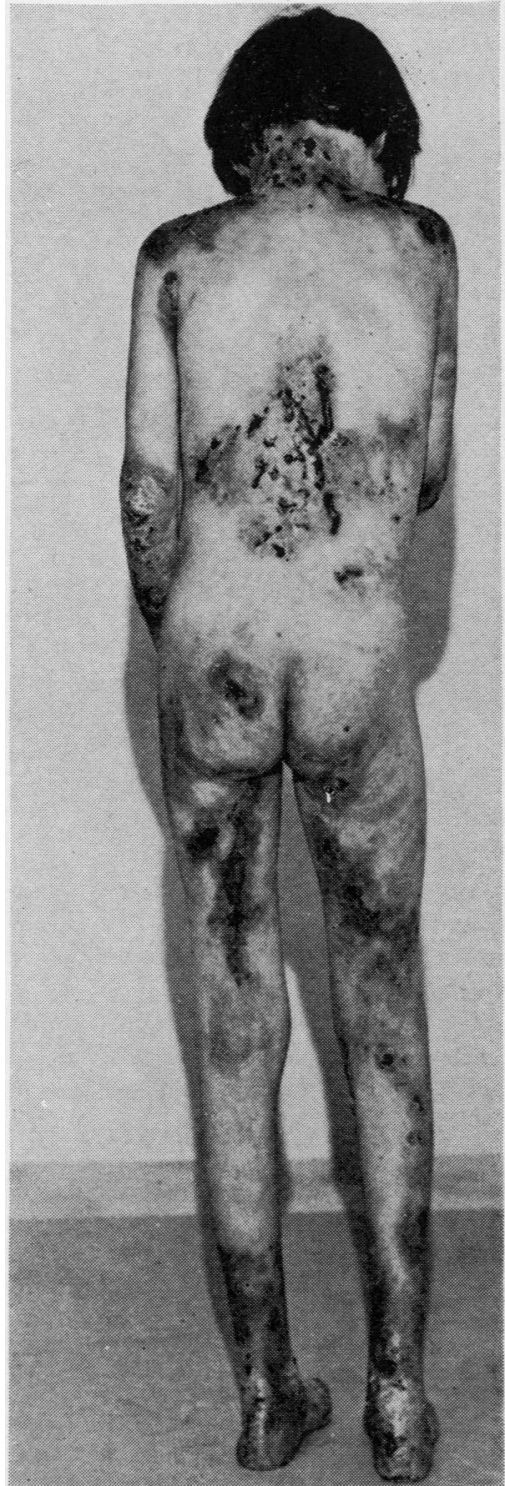

Fig 1 Appearance of patient at age 12 years. Bullae, ulcers, and scars are evident.

resected near its extreme proximal and distal ends. Inspection of the specimen revealed a very tight stricture in the upper third, and in this region there was an apparent perforation which had entered the wall of the oesophagus and dissected the muscle layers down its entire length. As a result, there were two lumina visible, the larger of which appeared to be the false lumen created by muscle separation. A cervical oesophagostomy of the remaining proximal

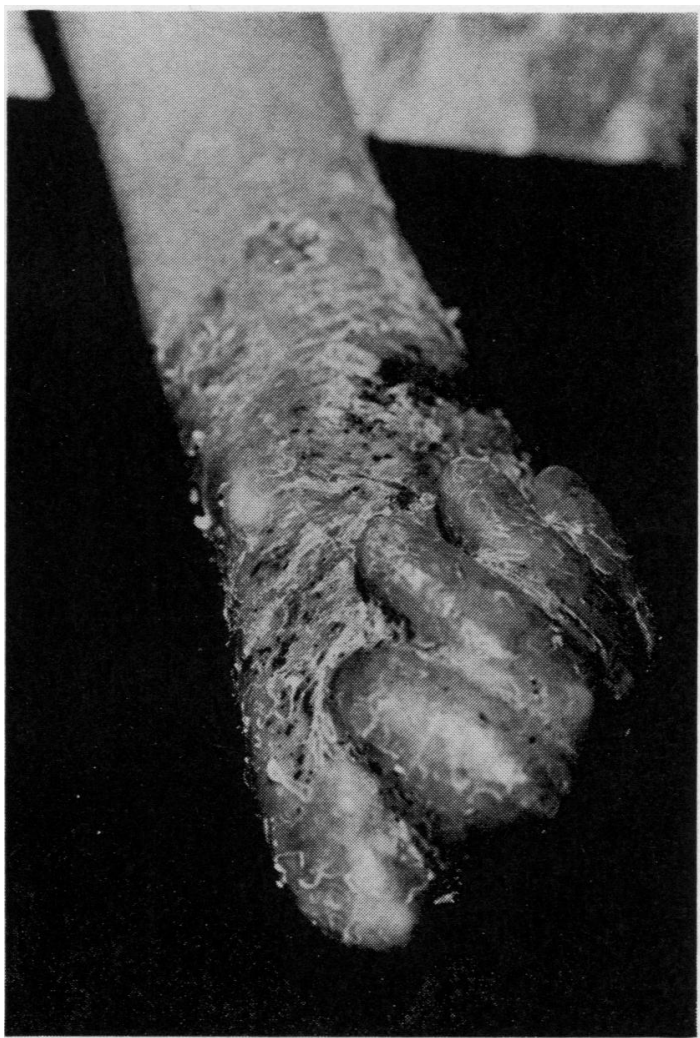

Fig 2 Appearance of hand at age 17 years showing developing mitten-type deformity.

oesophageal stump, feeding gastrostomy, and pyloromyotomy, were also performed. The patient's recovery was uneventful, and she was discharged 15 days later.

\section{Pathological findings}

The resected segment of oesophagus was $10 \mathrm{~cm}$ in length. The thin wall of the false lumen, which had been opened, partly surrounded the true oesophagus. This area was tan coloured and had a rough, transversely corrugated surface which probably corresponded to bundles of circular smooth muscle. The proximal $5 \mathrm{~cm}$ of true oesophagus were eroded and communicated with the false lumen on one side (fig 3); distally, the true oesophagus had a firm, thickened $(3 \mathrm{~mm})$ wall and a narrow lumen $(1.2 \mathrm{~cm})$. The mucosa of the most proximal, dilated end of the specimen $(3.5 \mathrm{~cm}$ circumference) had a nodular appearance. A shaggy exudate was present on the external aspect of the specimen.

On light microscopy, there was extensive 


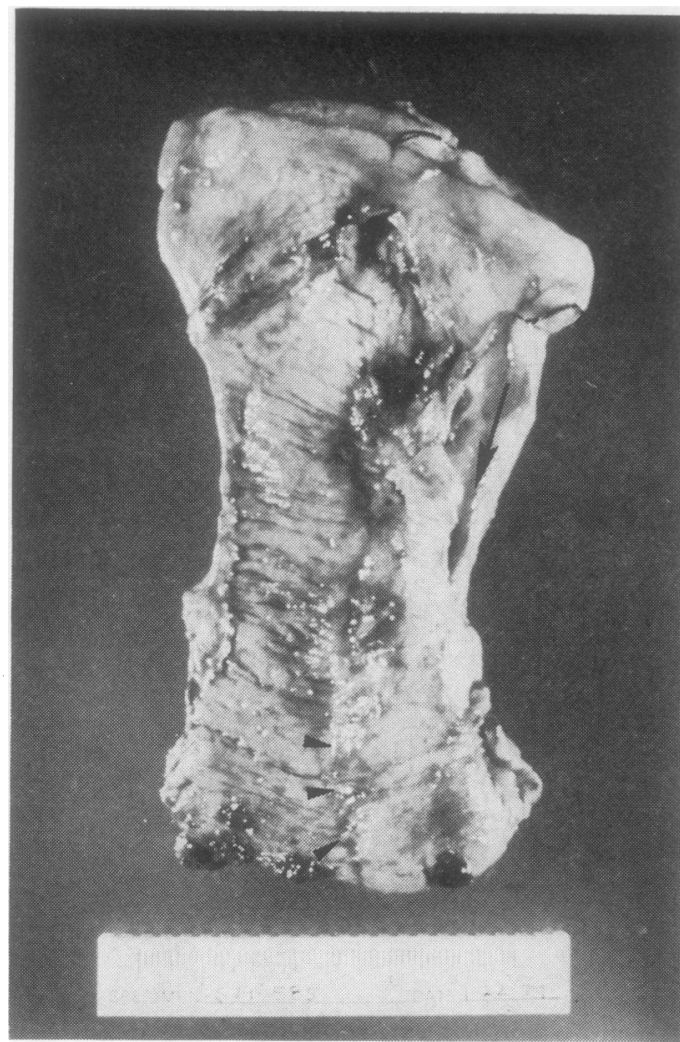

Fig 3 Oesophageal stricture with partially opened true lumen (large arrow) and exposed false lumen showing one lateral plane of cleavage (small arrows at distal end).

ulceration of the proximal oesophagus with submucosal fibrosis. Focal collections of plasma cells and lymphocytes were present in the muscularis. The plane of dissection, which was identified by an acute inflammatory exudate, varied in position between the muscularis mucosa and the layer of circular muscle or between the latter and the outer longitudinal muscle layer (fig 4). Gas cysts were seen in the plane of dissection and in the fibrinopurulent exudate which surrounded the oesophagus. Cardiac-type mucosa with numerous goblet cells and a villonodular pattern lined the proximal oesophagus where focal ulceration was seen. In some areas, islands of normal squamous epithelium were admixed with the cardiac-type mucosa (fig 5). Gastric mucosa composed of cardiac glands containing groups of parietal and chief cells was present at the distal end of the resected specimen. Blocks of tissue from the proximal oesophagus were processed in the usual manner for electron microscopy. Separation of

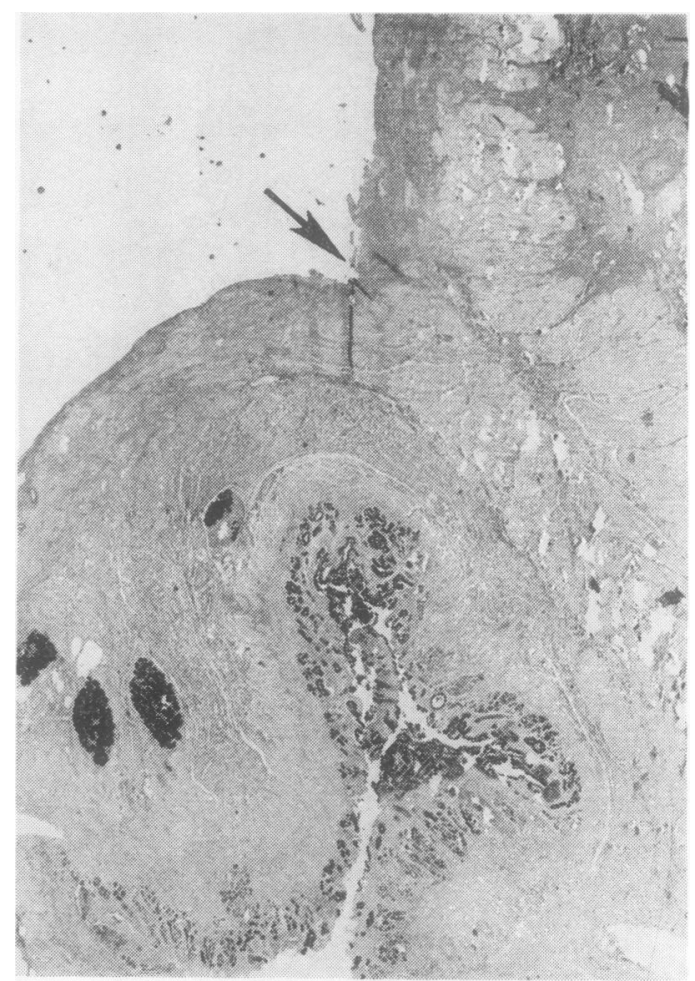

Fig 4 Section of distal oesophagus showing plane of cleavage through muscularis (arrow) and adjacent true oesophageal lumen lined by gastric-type mucosa. Periodic acid-Schiff, original magnification $\times 4$.

the epithelium from the underlying mucosa at the sub-basal laminar level was seen in one area.

In summary, there was (1) deep and extensive ulceration of the oesophagus which was lined proximally by columnar epithelium-that is, cardiac-type glands, (2) dissection between muscular layers, and (3) acute mediastinitis.

\section{Discussion}

Epidermolysis bullosa is a rare skin disease manifested by the appearance of bullae on minor $\omega$ mechanical skin traumatisation. There are scarring and non-scarring forms, and each may $\stackrel{\varrho}{\simeq}$ be inherited in a dominant or recessive way. ${ }^{2}$ In $\mathbb{D}_{\overparen{D}}$ the recessive form of epidermolysis bullosa dystrophica, as expressed in our patient, evidence of the disease begins at, or soon after, birth. Bullae may involve skin, mucous membranes, and other organs lined by stratified squamous $\triangle$ epithelium, such as the oesophagus and anus. Bullae frequently progress to erosions and ulcers 


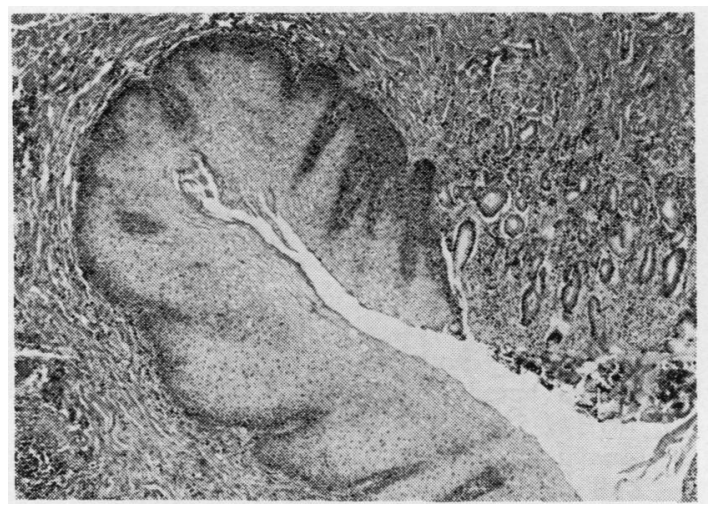

Fig 5 Section of oesophagus showing oesophageal mucosa beside gastric-type mucosa. Note separation of squamous epithelium from submucosa near the junction (centre). Haematoxylin and eosin, original magnification $\times 40$.

that heal slowly with scarring, and this results in loss of hair and nails and in clublike deformities of the hands and feet. An occasional late complication is the development of squamous cell carcinoma. ${ }^{34}$

Only patients with the recessive form develop oesophageal involvement. The friable oesophageal mucosa is readily injured by solid or hot foods with resultant bulla formation and occasional ulceration. The common sequela, caused by repeated injury, is the development of scars and strictures with a clinical picture of dysphagia that usually begins in the first decade. Blistering of the mouth and pharynx leads to contractures that limit the ability to open the mouth. Radiological examination of the oesophagus usually shows a single or more rarely, a few areas of annular stenosis, most often in the cervical portion. ${ }^{5}$ Orlando et $a l^{8}$ stated that $50 \%$ of strictures occur in the upper one-third of the oesophagus, $25 \%$ in the lower one-third, and $25 \%$ in multiple sites. Diffuse involvement, as demonstrated by our case, is distinctly uncommon. ${ }^{5}$ Nix and Christianson, ${ }^{1}$ in their survey of 24 cases of oesophageal involvement in epidermolysis bullosa to date, found only one case $^{7}$ in which the entire oesophagus was narrowed. In most cases, the stenotic segments vary from two to four and a half $\mathrm{cm}$ in length. Most patients who develop strictures remain relatively stable if maintained on a pureed diet, and radiological examination does not reveal progression of established strictures. ${ }^{6}$ Perforation may occur rarely, as in our case. Nix and Christianson $^{1}$ reported the death of a patient as a result of rupture of the distal one-third of the oesophagus.

Our case is very unusual, not only because the total oesophagus was involved throughout and perforated, but also because the ulceration dissected and produced a "double-barrel" deformity of the oesophagus which structurally, is strictly analogous to double-barrel formation in aortic dissection with re-entry. The acquired fibrous encasement of the oesophagus was probably sufficiently dense to prevent anything more than the pinhole rupture all the way through to the thoracic cavity, which was found at thoracotomy. However, ulceration also occurred to the level of the plane of cleavage between smooth muscle layers, and this compounded with the proximal pressure of deglutition, is the most likely aetiology of the subsequent dissection.

Histologically, in the dystrophic scarring form, there is a clean separation between the epithelial basement membrane and the underlying dermis, the basement membrane remaining in contact with the basal layer of epithelial cells. ${ }^{8}$ In contrast, in the nonscarring form, separation occurs between the basal epithelial cells and the basement membrane. This is well illustrated by the ultrastructural study of our case. Pearson et $a l^{8}$ believe that the marked reduction of fine fibres ("anchoring fibrils") normally found on electron microscopy beneath the basement membrane, is responsible for the separation. Briggaman and Wheeler ${ }^{9}$ described an absence of anchoring fibrils and also found them to be absent in non-traumatised skin. Using recombinant grafts of normal skin and skin affected by epidermolysis bullosa that were subsequently co-cultured on chorioallantoic membranes of embryonated chicken eggs, they were able to demonstrate that the anchoring fibrils are products of a normal dermis but fail to be formed by the defective dermis of epidermolysis bullosa.

We suggest that in our case, the presence of gastric mucosa in the upper oesophagus represented metaplasia during epithelial repair, implying the presence of a versatile pluripotent stemcell, either in the epithelium or in oesophageal glands. However, it is impossible definitely to exclude pre-existent ectopic gastric mucosa which could also be responsible for ulceration. Columnar epithelium has been reported once ${ }^{10}$ in epidermolysis bullosa of the oesophagus, and in this instance it was attributed to fibrosis with tractional herniation of gastric mucosa. Continuity with gastric mucosa in our case could not be excluded because of extensive ulceration. However, its presence, extending up 
to this high level, would be unlikely. To our knowledge, the case we have presented is the first report of the development of columnar epithelium in association with epidermolysis bullosa of the oesophagus.

\section{References}

1 Nix T, Christianson H. Epidermolysis bullosa of the esophagus. South Med J 1965; 58:612-20.

2 Pearson R. Studies on the pathogenesis of epidermolysis bullosa. J Invest Dermatol 1962; 39:551-75.

3 Naef A, Savary M, Ozzello C, Pearson F. The columnar-lined lower esophagus: an acquired lesion with malignant predisposition. J Thorac Cardiovasc Surg 1975; 70:826-35.

4 Ackermann A. Histologic diagnosis of inflammatory skin diseases. Philadelphia: Lea and Febiger, 1978: 583-8.

5 Adler $R$. The lower esophagus lined by columnar epithelium: its association with hiatal hernia, $\mathbb{D}$ ulcer, strictive, and tumour. $J$ Thorac Cardiovasc Surg 1963; 45:13-34.

6 Orlando R, Bozymski E, Briggaman R, Bream $\vec{\circ}$ C. Epidermolysis bullosa, gastrointestinal manifestations. Ann Intern Med 1974; 81:203-6. $\vec{\omega}$

7 Steurer A. Ueber beteiligung der schleimhaut des mundes und der speiseroehre bei epidermolysis $\vec{x}$ bullosa herediaria. Arch Ohr Nas Kehlkopfheilk $\dot{\omega}_{j}$ 1921; 108:11 (as quoted by Nix and Christianson $^{1}$ ).

8 Pearson R, Potter B, Strauss F. Epidermolysis bullosa hereditaria letalis. Arch Dermatol 1974; 109:349-55.

9 Briggaman, R, Wheeler C. Epidermolysis bullosa dystrophica recessive: a possible role of anchoring fibrils in the pathogenesis. $J$ Invest $\overparen{\curvearrowright}$ Dermatol 1975; 65:203-11.

10 Alpert M. Roentgen manifestations of epidermolysis bullosa. $A J R$ 1957; 78:66-72. 